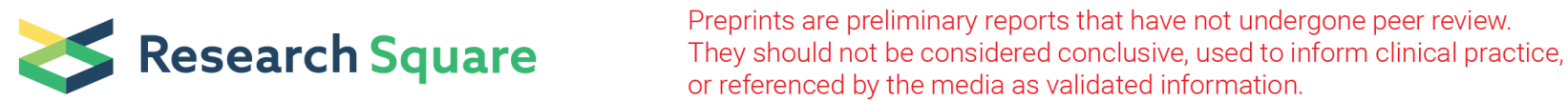

\section{Prediction of maxillary canine width by maxillary and mandibular central incisors by the aid of an equation as a preventive measure}

Fadil A Kareem ( $\boldsymbol{\sim}$ fadil.kareem@univsul.edu.iq )

\section{Research article}

Keywords: Prediction, Maxillary permanent canine width, maxillary and mandibular central incisors, prediction equation, mixed dentition period

Posted Date: February 3rd, 2020

DOI: https://doi.org/10.21203/rs.2.22449/v1

License: (c) (1) This work is licensed under a Creative Commons Attribution 4.0 International License.

Read Full License 


\section{Abstract}

Background: Mixed dentition analysis is critical for early diagnosis of malocclusion because many malocclusions appear in this period. The aim of the present study is finding out specific prediction equation for estimation of mesio-distal width of maxillary unerupted canine at mixed dentition period depending on the width of upper and lowers central incisors.

Materials and methods: Hundred orthodontic patients' (50 males and 50 females) study casts visited a private clinic in Sulaimani City/Iraq were randomly selected and the largest mesio-distal dimensions of maxillary and mandibular central incisors and maxillary canines were measured by a digital calliper. The age range of patients was 14-24 years according to the inclusion criteria. Correlation and linear regression tests were applied on the collected data to find a prediction equation to estimate the mesiodistal width of maxillary permanent canine at early mixed dentition period relying on mesio-distal width of either mandibular or maxillary permanent central incisor.

Results: Correlation coefficients of maxillary and mandibular central incisor were 0.664 and 0.639 , respectively. It indicates a moderately strong correlation between the width of maxillary and mandibular central incisors and maxillary canine width. Establishment of valid practical prediction equations, one for maxillary central incisors (1.205+ $0.796 \times$ Maxillary central incisor width) and the other for mandibular central incisors (1.205+1.205 x Mandibular central incisor width) with the former one being more reliable than the second one have a significant influence on construction of treatment plan at mixed dentition period.

Conclusion: Moderately strong prediction equation is established to the mesio-distal width of maxillary permanent canine prior to their emergence in the oral cavity as an attempt to prevent their impaction or malposition.

\section{Background}

Mixed dentition analysis is critical for early diagnosis of malocclusion because many malocclusions may emerge in this period, in the age range of 6 to 12 years, so a proper intervention at this time may be able to prevent or at least minimize the severity of these malocclusions [1]

Space analysis implies preventive measures, which are important to prevent potential irregularity from progressing into a more severe malocclusion [2]. Estimating the mesio-distal width of the unerupted canines and premolars according to the size of erupted teeth (central incisors and first molars) can lead to earlier diagnosis of malocclusion [3]. These analyses have to be simple, quick with minimum error, should not necessitate any special tool, and must be specific for each arch [2].

Three techniques have been tried to estimate the mesio-distal dimensions of unerupted premolars and canine crowns: (1) measurements from erupted teeth, (2) measurements from radiographs, and (3) a combination of measurements from erupted teeth and from radiographs of unerupted teeth [4]. 
Measurements from radiographs require time, specific equipment, and are less practical as undistorted radiographic images are required; moreover, the radiation burden is always unjustifiable [5].

The Moyers mixed dentition analysis which is the oldest one depends on a correlation of tooth size; measurement of one tooth or a group of teeth in order to predict the size of the other teeth accurately in the same oral cavity. The mandibular incisors have been chosen for measurement to estimate the size of the upper and lower posterior teeth due to their early eruption in the mixed dentition and accurate measurements [5]. Both Moyers analysis and Tanaka and Johnston prediction method are not considered as an accurate when applied to a sample of Sulaimani population as illustrated by a previous study in Sulaimani city [6].

The maxillary permanent canine behaves as the cornerstone of occlusion and has a key role in smile design [7]. Furthermore, upper canines tend to erupt later in the sequence of the normal eruption of the upper teeth [8] andalmost all authors approved that the maxillary canine follows a longer tortuous eruption path than any other tooth. However, the length of its path of eruption and the magnitude, as well as the timing of the changes in direction does not seem to be quantified [9]. No study till now conducted among Sulaimani population to determine mesio-distal width of unerupted maxillary canine alone not in combination with unerupted canine and premolars. Accordingly, the aim of the present study is finding out specific prediction equation for estimation of mesio-distal width of maxillary unerupted canine at mixed dentition period depending on the width of upper and lowers central incisors as a preventive measure of expecting the future impaction or malocclusion in a sample of Sulaimani population.

\section{Methods}

This cross-sectional study was conducted to find out special prediction equation for maxillary canine crown width estimation based on the width of each of maxillary and mandibular central incisors.

Ethical approval from the ethical committee of the medical colleges/university of Sulaimani was obtained. The sample of the study comprised of randomly selected 120 dental orthodontic patient models at the age range between 13-24 years old who were visited private clinics in Sulaimani city. The purpose of the study was explained to the participants and/or their parents in case of nonadult patients and a consent to participate in the study was provided by the patients and/or their parents.

The participants were encompassing the inclusion criteria of the study which was: complete set of permanent dentitions in the jaws, no interproximal caries and restoration, no previous history of orthodontic treatment, no congenital anomalies without attrition occlusally and interproximally.

The largest mesiodistal width of the mandibular and maxillary central incisors, canines were measured using a digital calliper (Mitutoyo, Tokyo, Japan) with $0.001 \mathrm{~mm}$ accuracy for each model. Intra-examiner and Inter-examiner calibration of the measurements were performed by re-measuring 20 random cases by the same examiner one week later and another examiner, respectively. Afterwards, the mean values were compared and analyzed statistically using paired t-tests. The collected data were subjected to SPSS 
computer program version 24 to find both descriptive and inferential analysis. A linear regression test was applied to attain equation for prediction of mesio-distal width of maxillary canine depending on mesio-distal dimensions of maxillary and mandibular central incisors.

Calculation of the obtained equation was carried out as; $y=a+b x$ where " $y$ " is the dependent variable, i.e. width of maxillary permanent canine, " $x$ " is the independent variable, i.e. sum of mandibular or maxillary central incisors, "a" is the y-intercept, and "b" is the slope of the regression.

\section{Results}

The average width of the right and left sides of the measured teeth was calculated and used in the study. Descriptive statistics of the measured teeth were presented in table (1). Inter and Intra examiner calibrations showed no statistical differences. Correlation between widths of maxillary permanent central incisors, mandibular central incisors with a maxillary permanent canine was calculated by SPSS computer software version 24 . Correlation coefficients of maxillary and mandibular central incisor were 0.664 and 0.639 , respectively (Table 2). Accordingly, a correlation test finding reveals that each of maxillary and mandibular central incisors can be used to predict the size of the maxillary permanent canine. However, the result with the use of maxillary central incisor was more reliable for this estimation.

The obtained equation was calculated as; $y=a+b x$ where " $y$ " is the width of maxillary permanent canine, " $x$ " is the sum of mandibular or maxillary central incisors, " $a$ " is the y-intercept, and "b" is the slope of the regression. Hence, the equation regression for prediction of maxillary permanent canine was as the followings:

\section{$y=a+b x$}

- Maxillary canine width $=1.205+0.796 \times$ Maxillary central incisor width

- Maxillary canine width $=1.205+1.205 \times$ Mandibular central incisor width

The obtained Coefficient of determinations $\left(r^{2}\right)$ was $(41 \%)$ and $(44 \%)$ for the use of mandibular and maxillary central incisors as predictors, respectively. $\mathrm{R}^{2}$ measures the proportion of variation in the dependent variable that can be attributed to the independent. The closer coefficient of determination to 1.0 means better explanation of upper canine width by central incisors.

\section{Discussion}

Size prediction of the unerupted teeth during the mixed dentition period is pivotal in the management of occlusion development of a growing person and it is of prime importance in constructing a perfect treatment plan [3]. Additionally, mixed dentition analysis permits tooth size-arch length discrepancy identification in an early stage and dictates various treatment opportunities as maintaining and regaining spaces maintenance, serial extractions, eruption guidance and observation with no intervention [10] 
The long eruption path of maxillary canine and very long time between its initial development and emergence in oral cavity considered it to be more affected by a deflection from its path of eruption. More precisely, it may be impacted, displaced buccally or palatally [11]. So picking up special attention to the maxillary canine is regarded as the utmost important factor in minimizing the occurrence of malocclusion or at least the decrease in its severity.

Most of the previous studies regarded the mandibular central incisor as a more reliable index for prediction of the size of unerupted permanent teeth as Tanaka and Johnston in 1974 [12], Moyers in 1988 [11] on Caucasian population, Al-Khadra in 1993 on Saudi Arabian population [13], Lee-Chan et al in 1998 on Asian-Americans [4], and Diagne et al in 2004 on Moroccan population [2]. In regard to the findings of the present study, the use of maxillary central incisors is more reliable than mandibular central incisors as a predictor of maxillary canine width.

Most of the methods have been established by conducting studies on the Caucasian ethnic group; therefore, they might not be accurate for other ethnicities [14]. Additionally, race-specific predictive data are needed for different parts of the world. No research has been carried out on the Kurdish population on the prediction of upper canine alone as a single tooth, not in a combination of canine and premolars. The only study on a Kurdish sample of Sulaimani population/Iraq was carried out in 2010 by Mahmood and Kareem concluded that Tanaka and Johnston prediction method was not accurate when applied to a sample of Sulaimani population. Furthermore, they applied an equation developed by Nourallah et al in 2002 [15] on a sample of Sulaimani population and proved the equation's accuracy and validity on a Kurdish sample. The current study takes into account the development of a prediction equation of upper canine width as a complementary study to the previous study.

\section{Conclusion}

Both maxillary and mandibular central incisors can be used as a predictor for estimation of mesio-distal width of maxillary unerupted canine without a radiograph. Maxillary central incisor considered to be more reliable. Moderately strong prediction equation is established to the mesio-distal width of maxillary permanent canine prior to their emergence in the oral cavity as an attempt to prevent their impaction or malposition.

\section{Declarations}

\section{Acknowledgements}

\section{- Not applicable.}

\section{- The author declares that he has no competing interests.}




\section{Abbreviations:}

- Not applicable.

\section{Ethics approval and consent to participate:}

- Ethical approval for the this research study is obtained from the ethical committee of medical colleges in the University of Sulaimani, Iraq.

\section{Consent for publication:}

- Verbal consent was obtained from all participants.

\section{Availability of data and materials:}

- The datasets used and/or analysed during the current study are available from the author on request.

\section{Funding:}

- This research did not receive any specific grant from funding agencies in the public, commercial, or not-for-profit sectors.

\section{References}

1. Ling JY, Wong RW. Tanaka-Johnston mixed dentition analysis for southern Chinese in Hong Kong. Angle Orthod. 2006;76(4):632-6.

2. Diagne F, Diop-Ba K, Ngom Pl, Mbow K. Mixed dentition analysis in a Senegalese population: Elaboration of prediction tables. Am J Orthod Dentofacial Orthop. 2003;124:178-83.

3. Bernabé E, Flores-Mir C. Are the lower incisors the best predictors for the unerupted canine and premolars sums? An analysis of a Peruvian sample. Angle Orthod. 2005; 75(2):202-7.

4. Lee-Chan S, Jacobson BN, Chwa KH, Jacobson RS. Mixed dentition analysis for Asian-Americans. Am J Orthod. 1998; 113:293-9. 
5. Proffit WR and Fields HW. Contemporary orthodontics, 3rd edition, Mosby Inc; 2000: 165-70

6. Mahmood T and Kareem F. mixed dentition analysis in a sample of Sulaimani population. KAJ. 2010: 1(7-8A):23-8

7. Raghoebar GM, Boering G, Vissink A, Stegenga B. Eruption disturbances of permanent molar: a review. J Oral Pathol Med. 1991; 20: 159-66

8. Quadras DD, Nayak US, Ravi MS, Pujari P. Early prediction of maxillary canine impaction using sectors and angular measurement - A radiographic study. MJDS. 2017; 2(2), 7-11.

9. Coulter $J$ and Richardson A. Normal eruption of the maxillary canine quantified in three dimensions. Eur J Orthod. 1997; 19: 171-183

10. Philip NI, Prabhakar M, Arora D, Chopra S. Applicability of the Moyers mixed dentition probability tables and new prediction aids for a contemporary population in India. Am J Orthod Dentofac. 2010; 138(3):339-45.

11. Moyers RE. Handbook of orthodontics. 4th ed., Chicago, IL, Year Book Medical Publishers Inc; 1988 : 235-9.

12. Tanaka MM, Johnston LE. The prediction of the size of unerupted canines and premolars in a contemporary orthodontic population. J Am Dent Assoc. 1974; 88(4):798-801.

13. Al-Khadra BH. Prediction of the size of unerupted canines and premolars in a Saudi Arab population. Am J Orthod Dentofac. 1993;104(4):369-72.

14. Abu Alhaija ES, Qudeimat MA. Mixed dentition space analysis in a Jordanian population: comparison of two methods. Int J Paediatr Dent. 2006;16(2):104-10

15. Nouralla $A B$, Khoraji $M N$ and Gesch D. New regression equations for predicting the size of unerupted canines and premolars in a contemporary population. The Angle Orthod. 2002; 72(3):216-21

\section{Tables}

Table (1): Descriptive statistics of the study sample

\begin{tabular}{|l|c|c|c|c|c|}
\hline \multicolumn{7}{|c|}{ Descriptive Statistics } \\
\hline \multirow{2}{*}{ Variables } & $\mathbf{N}$ & Minimum & Maximum & Mean & S.D. \\
\hline Age & 100 & 14.00 & 24.00 & 17.20 & 2.391 \\
\hline Maxillary central incisors & 100 & 7.47 & 10.35 & 8.900 & .599 \\
\hline Mandibular central incisors & 100 & 4.80 & 6.42 & 5.580 & .358 \\
\hline Maxillary canines & 100 & 7.14 & 9.33 & 8.088 & .514 \\
\hline
\end{tabular}

Table (2): Correlations between dependent and independent variables 


\begin{tabular}{|l|l|c|c|}
\hline \multicolumn{2}{|l|}{} & Maxillary central incisor** & Mandibular central incisor** \\
\hline \multirow{2}{*}{ Maxillary canine width* } & Pearson Correlation & .667 & .639 \\
\cline { 2 - 4 } & Sig. (2-tailed) & .000 & .000 \\
\cline { 2 - 4 } & r & 0.444 & 0.408 \\
\hline & N & 100 & 100 \\
\hline
\end{tabular}

*dependent variable

**independent variables 